\title{
An Observation of an Inverted Type U Solar Burst Due to AR1429 Active Region
}

\author{
Z. S. Hamidi ${ }^{1, *}$, N. N. M. Shariff ${ }^{2}$, C. Monstein ${ }^{3}$ \\ ${ }^{1}$ School of Physics and Material Sciences, Faculty of Sciences, MARA University of Technology, \\ 40450, Shah Alam, Selangor, Malaysia \\ ${ }^{2}$ Academy of Contemporary Islamic Studies (ACIS), MARA University of Technology, \\ 40450, Shah Alam, Selangor, Malaysia \\ ${ }^{3}$ Institute of Astronomy, Wolfgang-Pauli-Strasse 27, Building HIT, Floor J, \\ $\mathrm{CH}-8093$ Zurich, Switzerland \\ *E-mail address: zetysh@salam.uitm.edu.my
}

\begin{abstract}
A detailed investigation of an inverted type $U$ solar burst in meter region and their associated the solar flare and Coronal Mass Ejections (CMEs) has been reported. Solar type U burst has been observed at the National Space Centre, Banting, Selangor detected by the Compound Low Cost Low Frequency Transportable Observatories (CALLISTO) system in the $150 \mathrm{MHz}$ till $400 \mathrm{MHz}$ at the low frequency band. An inverted U type is occurring on 9th March 2012 between 4:00 UT to 4:15 UT within 1 minute (4:12 - 4:13) UT. From the dynamic spectra of CALLISTO, we have identified metric type U burst with maximum emission near the frequency $385 \mathrm{MHz}$. In specific, the continuum type III burst will soon structure this burst due to our observations. Other types such as type II and IV are only appearing only after type $U$ burst is ejected and appear at the same point of the solar flare event. Since the $U$ burst activity coincides with the peak of the hard X-ray flare at 4:12UT in AR1429, we classified that the event is associated with the injection of the high energetic particles. In conclusion, it is confirmed that an inverted type $\mathrm{U}$ burst is initiated after a complex and a group solar radio burst type III.
\end{abstract}

Keywords: CALLISTO; low frequency; solar burst, type U; Radio Frequency Interference (RFI)

\section{INTRODUCTION}

Solar radio type $U$ bursts with a change of the frequency drift sign are observed in a wide frequency band from decimeter to decameter waves [1-3]. The first observation of inverted type $U$ burst has been reported by [4]. Among the various impulsive of solar burst, type $U$ is a unique and interesting to study in detail in terms of association with solar flares. It is well understood that type $U$ burst is similar with a type III burst except the excited by electrons propagating in a magnetic arch [5]. Based on previous studies, it occurs during the large flare, but still much more rarely and basically generated to invert of the $\mathrm{J}$ burst [6]. There is evidence that showed the most regions this type happen is between 20 to $200 \mathrm{MHz}$ in asymmetrical form [7]. Until now, the mechanism of generation of type $U$ bursts is still a matter of ongoing study. It was observed that in some cases, it will form in fundamental or 
harmonic structure, but the configuration is very rare. Many theories have been proposed to explain type U burst. Theoretically, there is an interpretation by [8] suggested that the electron beams propagating in closed coronal loops requires (i) a high ambient temperature in the relevant coronal loops which approximately $\mathrm{T} \geq 7 \times 10^{6} \mathrm{~K}$ and (ii) the magnetic strengths should be higher compared with those implied by potential explorations. Electron beams travelling through the solar corona excite 'Langmuir waves' near the local electron plasma frequency. When these Langmuir waves are converted into electromagnetic waves, the radiation can be detected in the radio region as bursts with very specific spectral signatures that depend on the magnetic structure which guides the beams. Normally, the ratio closed to open magnetic field structures increase with lower altitude [4]. Interestingly, it does not appear at a constant frequency, but regularly decreasing turning frequency [7].

The purpose of this work is to study solar burst type $U$ that leads the understanding of the relationship between other solar bursts that also occur at the same events. We want to relate the configuration of the magnetic field where they occur. In order to understand the characteristics of this burst, we analysed our data obtained by using the Compound Low Cost Low Frequency Transportable Observatories (CALLISTO) spectrometer. Present paper shall review in detailed regarding to the observation of an inverted type $U$ solar burst observed on $9^{\text {th }}$ March 2012 successfully observed [9]. In section 2, we describe on the CALLISTO system and experimental setup. The results are explained in section 3 and we discussed in detail in section 4.

\section{EXPERIMENTAL}

The first steps of solar radio burst research in Malaysia have been started since 2011 by collaborating with an e - CALLISTO network [10]. We started our project by constructing the Log Periodic Dipole Antenna (LPDA) which covered the range of frequency from $45 \mathrm{MHz}$ till $870 \mathrm{MHz}$ [11]. The CALLISTO system consists of the Log Periodic Dipole Antenna (LPDA), a preamplifier, and the spectrometer, which operating from $45 \mathrm{MHz}$ to $870 \mathrm{MHz}$ setup it at National Space Centre, Banting, Selangor since $22^{\text {nd }}$ February 2012. It is located at $\left(\mathrm{N} 02^{\circ} 49.488^{\prime} \mathrm{E} 101^{\circ} 36.168^{\prime}\right)$ where this site is mainly purpose for development of space and radio monitoring [12]. It consists the CALLISTO (Compound Astronomical Low-cost Low-frequency Instrument for Spectroscopy in Transportable Observatory) spectrometer, designed and built by electronics engineer Christian Monstein of the Institute for Astronomy of the Swiss Federal Institute of Technology Zurich (ETH Zurich), record the intensity of electromagnetic radiation at radio frequencies, between 45 and $870 \mathrm{MHz}$ [13]. Within this range, it could be possible to detect solar activities specifically solar flare and Coronal Mass Ejections (CMEs) [14]. We could observe started from 23.30 UT till 11.30 UT in daily basis. It covered approximately 12 hours monitoring. Up to date, there are 19 CALLISTO sites that actively provided a daily data. The channel resolution of this spectrometer is $62.5 \mathrm{kHz}$; while the radiometric bandwidth is about $300 \mathrm{KHz}$ with sampling time is exactly 1.25 milliseconds per frequency-pixel. In principle, data that obtained from the CALLISTO can be transferred to a computer and saved locally via a R232 cable in FIT files [17-26]. 


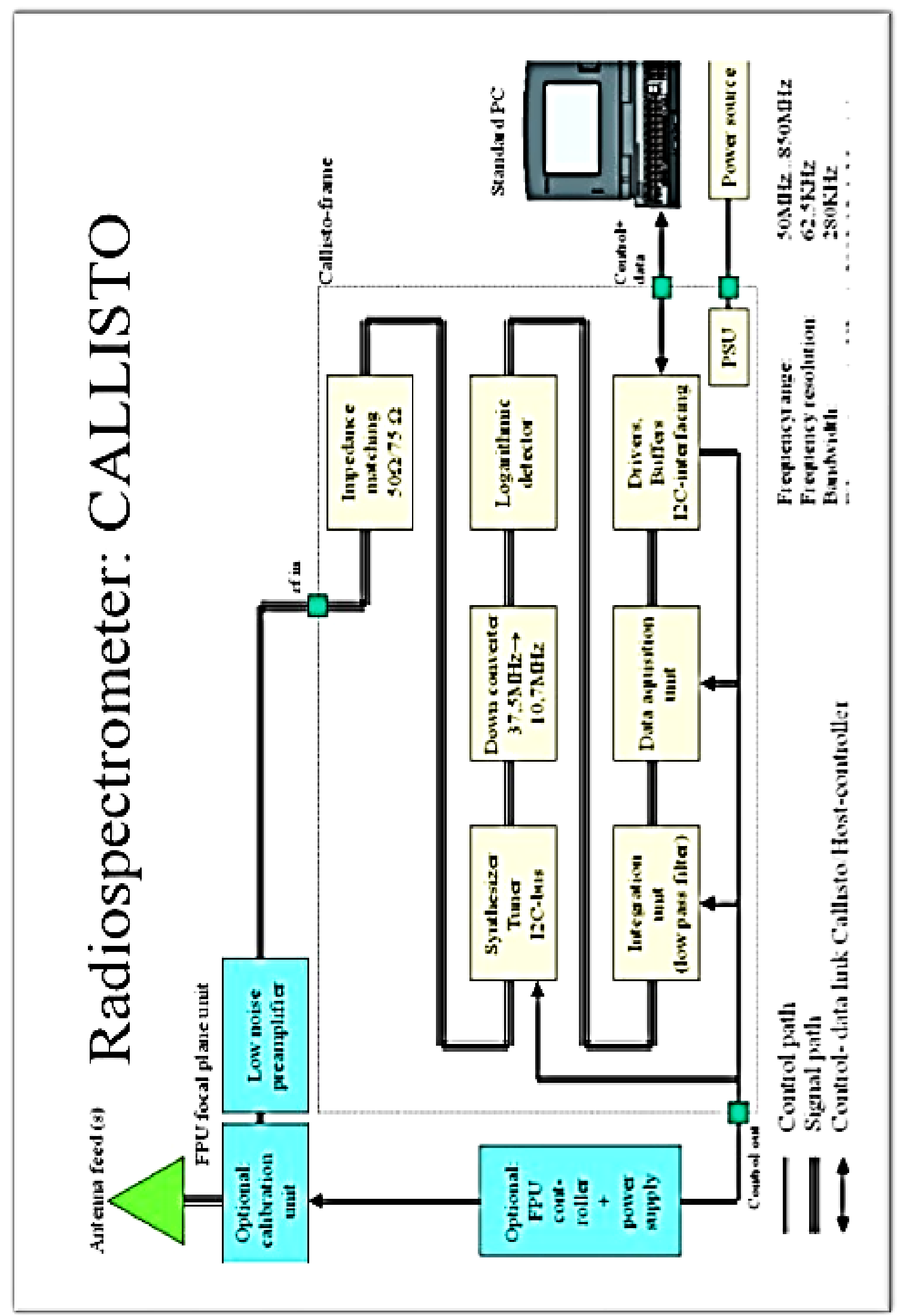

Figuire 1. Schematic diagram of the CALLISTO system. 
Current solar observatories, it provides a wealth of data at high spectral, temporal, and spatial resolutions with the specification of the 0.25 second time resolution. All data are stored with a scale factor and an offset applied so that the measured ADC digits range fits into the byte data range (0-255) per pixel. The antenna has a boom length 5.45 meters with gain $7 \mathrm{dBi}$ [15]. Meanwhile, Low Noise Preamplifier is connected to maximize a gain more than $10 \mathrm{~dB}$. Within this range, it could be possible to detect solar activities specifically the solar flare and the Coronal Mass Ejections (CMEs). This system has been successfully installed at the National Space Centre; Banting Selangor located $\left(3^{\circ} 5^{\prime} 00^{\prime \prime} \mathrm{N} 101^{\circ} 32^{\prime} 00^{\prime \prime}\right.$ E). Daily observations were performed for almost 12 hours. Schematic diagram of CALLISTO system is illustrated as in Figure 1. Up to date, the system which objectively was designed to observe solar flares in 24 hours monitoring has been distributed to 19 sites.

Our strength is that we can observe the solar activity within 12 hours throughout a year [16]. In a preliminary analysis, we have subtracted the pre-burst visibilities from the CALLISTO data in order to remove the signal from a quiet Sun. We also have checked the flux calibration by comparing the CALLISTO and relative amplitude of solar U burst. In this work we analyze the data obtained from our site using the CALLISTO spectrometer. We focused monitoring solar burst in the low frequency region. These data include one of the largest solar flare occurred since five years.

\section{OBSERVATION OF TYPE U BURST}

The observations were performed with the Log Periodic Dipole Antenna (LPDA) and CALLITO spectrometer that covered a broadband in the low frequency radio region. For the spectra described in this paper, a complete wavelength was accomplished every 15 minutes. Due to constrain of the interference factors, the range of $150 \mathrm{MHz}$ till $400 \mathrm{MHz}$ had been chosen as selected data. In particular, it has been observed that this burst occurs in the range of $310 \mathrm{MHz}$ till $384 \mathrm{MHz}$. It decreases with decreasing frequency, and this is readily understood by the fact that closed magnetic structures become rarer at increasing altitude. It drifts towards low frequencies. In this section we present the results of $U$ burst associated with another type bursts. The details of the spectral overview of ANGKASA, Banting, Selangor are illustrated in Figure 2.

The illustrations of such bursts are given in the dynamic spectrum in the left-hand panel of Figure 1. In normal case, $U$ burst that is reversed because the representation used here the frequencies are decreasing from bottom to top. During 9th March 2012, a strong M 7.9 solar flare was recorded from solar Active Region 1429. It is clearly seen an inverted type occurs between $310 \mathrm{MHz}$ till $384 \mathrm{MHz}$ within 1 minute. At this stage, electrons traveling to the top of the loop generate the branch of the type $U$ burst with negative frequency drift, whereas those traveling from the top generate the branch with the positive drift. It was found that M6.3 solar flare occurred in the active region AR 1429 starting at 03:32 UT and ending at 05:00 UT, with the peak at 04:12 UT. Associated with this event was type II with velocities 1285 $\mathrm{km} / \mathrm{s}$ and type IV radio sweeps along with a full halo CME first seen in SOHO/LASCO C2 imagery at 09/0426Z. Since the U burst activity (4.12-4.13 UT) coincides with the peak of soft X-ray flare (4:12 UT) in AR1429, we associate the region of the radio emission with the injection of the energetic particles. There is also a halo of Coronal Mass Ejections (CMEs) produced shortly after, and is expected to deliver a glancing blow to the earth's magnetic field sometime March 16th. All these results will be discussed in the next section. In the present case, it is proved that $\mathrm{U}$ burst occurs as isolated form. 


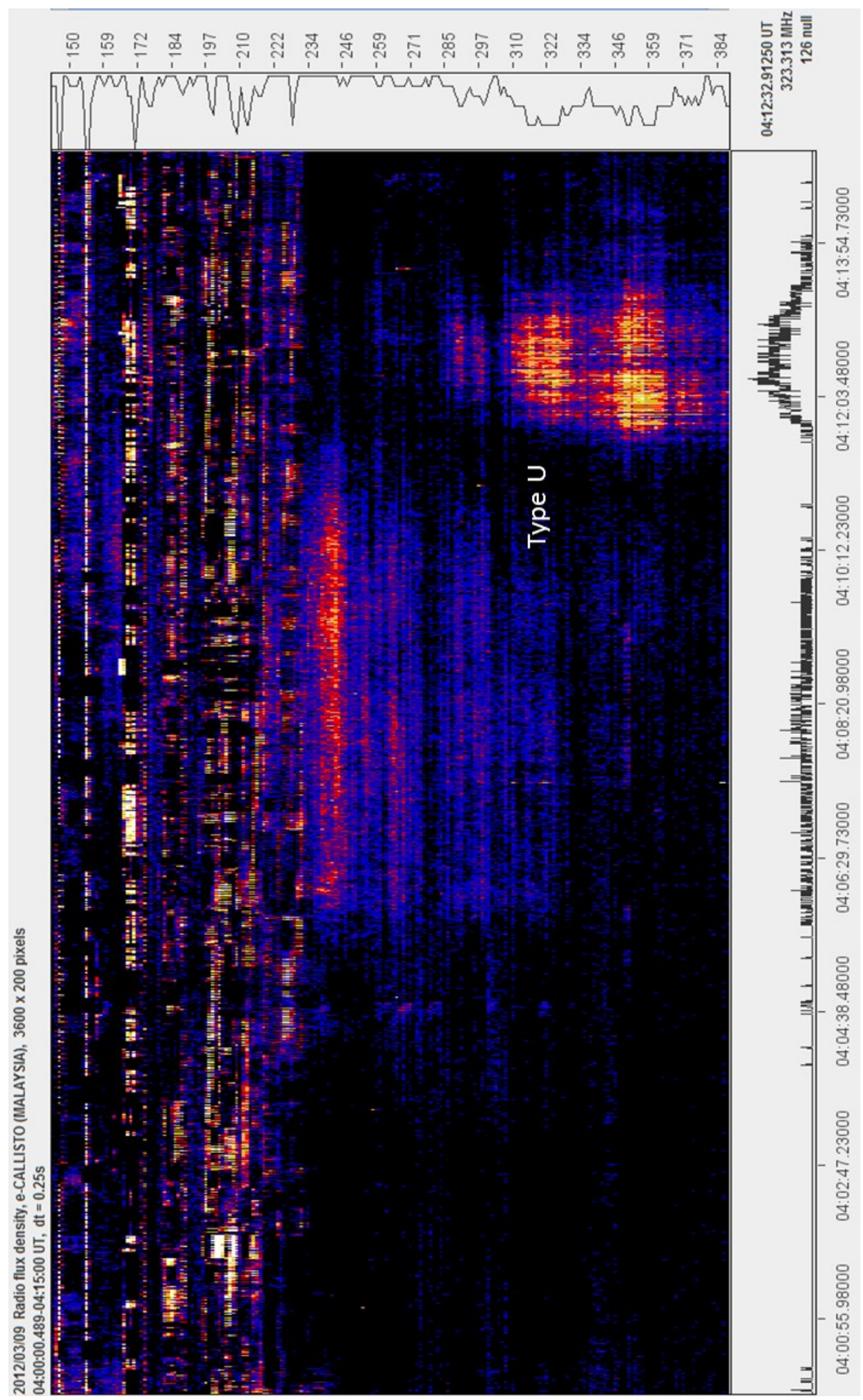

Figure 1. Spectrometer CALLISTO identifies an inverted type U solar burst at 4.12-4.13 UT. 
It looks very thin and believed to decrease with a rate typical of coronal transient speeds. The only indication of the type U burst is the continuum of type III burst before it happens. We could say that both types generated by a same storm. One could see from the Figure 2 is that a continuum type III burst dominated in the range of $234 \mathrm{MHz}$ to $259 \mathrm{MHz}$.

One common criterion for this unique type to form in decimetric range is that there is a type III storm activity before the evolution of $U$ burst. The observation support the idea that type U can be associated with type III at metric and longer wavelengths are only observed in transient magnetic loops associated with coronal mass ejections (CMEs). We also found that it followed by lanes of a type II burst (4:23-4:28) UT associated with a coronal shock wave.

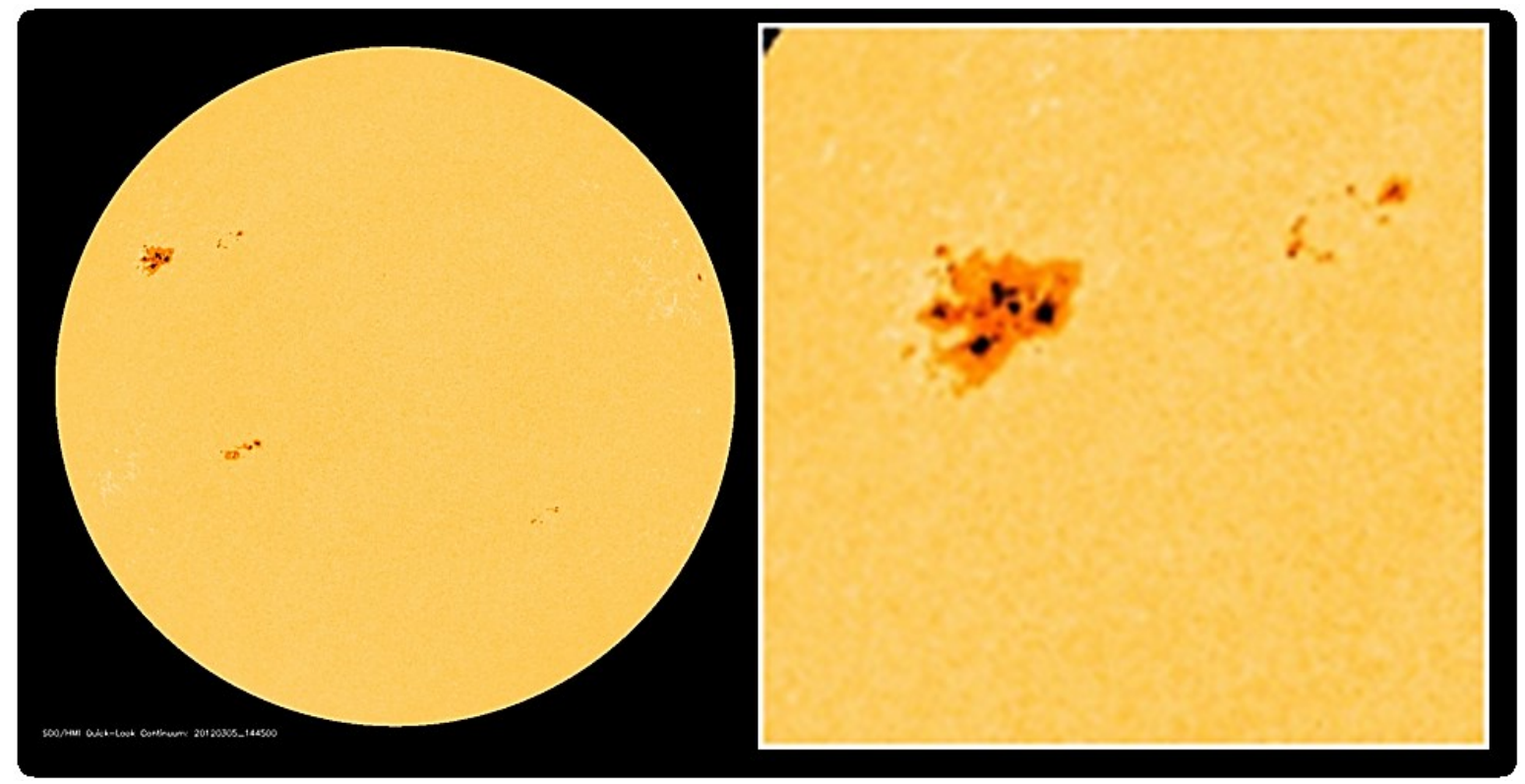

Figure 2. Image from the Helioseismic and Magnetic Imager (HMI) on the Solar Dynamics Observatory (SDO) shows active region 1429 in the upper left corner. The right figure is the enlarge image of AR1429. Credit to NASA/SDO/HMI.

Because the observation of $U$ type is the first report from our site, it is of interest to understand the chronology of this event. Our understanding of solar burst phenomena is still very limited and here we shall discuss the possible ways in which the emission could be generated. In order to understand the possible mechanism of dynamical structure of the burst, we have investigated relationships and time integrations of the bursts with sunspot active region 1429. It is well known that this region the source of many strong flares and geomagnetic storms and rotates into view on March 2nd, 2012. It produced two M-class flares and one the X-class so far. The M-class flares erupted on March 2nd and on March 4th. The third flare, rated an X1, peaked at 10:30 ET on March 4. On 9th March 2012, the sunspot number exceeds to 86 . This number represents that solar activities are active. Based on the GOES X-ray data, solar flare type M6.3 could be observed starting from 03:53 UT and continuously trigger within 24 hours. Moreover, type C9 has been detected at 2:25 UT and long lasting for six hours. This flare also followed by Coronal Mass Ejections (CMEs) confirmed the fact that this active region is still off to the side of the Sun, they will likely have a weak effect on Earth's magnetosphere. Figure 3 shows the active region 1429 recorded on 2nd March 2012 by the Solar Dynamics Observatory (SDO). Let us consider the chronology 
of radio emission during this event. It is expected that the AR1429 will active within 3 - 5 days started from 2nd March 2012, the first day where we could observe this region. The detection of solar bursts event has been observed on 9th March 2012 (about 3:32 UT till 5:00 UT) which is approximately occurring in 1 and half hour (88 minutes). The magnetic island formation and coalescence instability reconnection regime are responsible to the impulsiveness of different bursts. In order to observe how type U burst occurs, we try to relate the type of burst that detected at an early stage and also in the post stage. From our observations the evolution of this burst due to other bursts formation is described in Table 1 .

Table 1. The evolution of $U$ burst due to other bursts formation.

\begin{tabular}{ccc}
\hline Time (UT) & $\begin{array}{c}\text { Duration } \\
\text { (Minutes) }\end{array}$ & Type \\
\hline $3: 41-3: 43$ & 2 & Beginning signal \\
$3: 43-3: 44$ & 1 & Single burst type III \\
$3: 45-3: 58$ & 13 & Continuum burst type III \\
$4: 12-4: 13$ & 1 & U burst \\
$4: 23-4: 25$ & 2 & emission) \\
$4: 22-4: 26$ & 4 & Type IV (moving) \\
$4: 22-4: 27$ & 5 & Sudden peak type \\
$4: 23-4: 28$ & 5 & Condinumental and Harmonics Type II \\
$4: 32-4: 44$ & 12 & Type IV (moving) \\
$4: 45-4: 46$ & 1 & \\
& &
\end{tabular}

It was observed that at the beginning signal, there is an indicator that strong burst will occur due to the strong signal. We can clearly observed a very complex and a group type III solar burst at 3:41 UT. The U burst is decreasing with time at 4:13 UT. Interestingly, although our wideband frequency is limited to $250 \mathrm{MHz}$, it is still possible to detect more than one type's burst. In order to understand the phase of each burst take their own place during this solar event, we illustrate the formation of other bursts before and after solar burst type $U$ as in Figure 1. At first stage of the event, there is a single burst type III been identified at 3:41 UT. The result shows that it is confirmed that type III bursts is responsible to potentially form U type burst. In specific, the continuum type III burst will soon structure this burst due to our observations. Other types such as type II and IV are only appearing only after type U burst is ejected. Since the U burst activity (4:12-4:13) UT coincides with the peak of hard X-ray flare (4:12UT) in AR1429, we classified that the region of the radio emission is associated with the injection of the energetic particles. We found that other types such as type II and IV are only appearing only after type $U$ burst is ejected and appear at the same point of the Coronal Mass Ejections (CMEs) event.

\section{CONCLUSION}

The solar flare occurred on 9th March 2012 and time coverage included the entire time coverage and substantial part of the decay phase of the flare. So far, we have studied a 
spectacular sequence of solar radio burst recorded in the frequency range $150 \mathrm{MHz}$ to 400 MHz. Yet, this work leads to a better understanding of the relationship between type II, III, and $U$ burst activity, the configuration of the magnetic field where they occur and their associated with transient events. From the dynamic spectra of the CALLISTO, we have identified metric type $U$ burst with maximum emission near the frequency $385 \mathrm{MHz}$. Further progress will likely require us to obtain other data for comparison purpose. There are many observations that related to type $U$ burst, but each event is definitely represented a different pattern of evolution, range of frequency and the impact of space weather. Therefore, we still need to study the evolution of this burst to better understand the behaviour of the Sun. However, it is confirmed that type $U$ burst is initiated after a complex and a group solar radio burst type III.

\section{ACKNOWLEDGEMENT}

We are grateful to the LASCO,SDO/AIA, NOAA and SWPC make their data available online. This work was partially supported by the FRGS (600 RMI/FRGS 5/3 2012) UiTM grants. Special thanks to the National Space Agency and the National Space Centre for giving us a site to set up this project and support this project. Solar burst monitoring is a project of cooperation between the Institute of Astronomy, ETH Zurich, and FHNW Windisch, Switzerland, MARA University of Technology and University of Malaya. This paper also used NOAA Space Weather Prediction Centre (SWPC) for the sunspot, radio flux and solar flare data for comparison purpose. The research has made use of the National Space Centre Facility and a part of an initiative of the International Space Weather Initiative (ISWI) program.

\section{BIOGRAPHY}

Dr. Zety Sharizat Hamidi is currently a lecturer and focused in Solar Astrophysics research specifically in radio astrophysics at the School of Physics and Material Sciences, Faculty of Sciences, MARA University of Technology, 40450, Shah Alam, Selangor, Malaysia. Involve a project under the International Space Weather Initiative (ISWI) and also a lecturer in School of Physics and Material Science, at MARA University of Technology, Shah Alam Selangor.

Dr. Nur Nafhatun Md Shariff is a senior lecturer at Academy of Contemporary Islamic Studies (ACIS), MARA University of Technology, 40450, Shah Alam, Selangor, MalaysiaHer current research is more on sustainability; environmental aspect. She is looking forward for cross-field research, i.e. solar astrophysics, light pollution measurement (mapping) and religious studies.

C. Monstein is a senior Engineer at Institute of Astronomy, Wolfgang-Pauli-Strasse 27, Building HIT, Floor J, CH-8093 Zurich, Switzerland and one of the researchers who initiated the CALLISTO system around the world.

\section{References}

[1] M. J. Aschwanden, Güdel, M., ApJ 401 (1992) 736-753.

[2] H. Aurass, Coronal Physics from Radio and Space Observations, in: I.G. Trottet (Ed.), Lecture Notes in Physics, Springer, Berlin, 1997.

[3] B. Kliem, M. Karlick'y, A. O. Benz, Astron. Astrophys. 360 (2000) 715-728.

[4] S. Maxwell, Thomas, Proc. I. R. E. 46 (1958) 142.

[5] M. R. Kundu, Solar Radio Astronomy, John Wiley, 1965.

[6] A. D. Fokker, Space Science Reviews 2 (1963) 70-90. 
[7] S. Suzuki, G. A. Dulk, Bursts of type III and type V, in: D.J. McLean, N.R. Labrum (Eds.), Solar Radiophysics: Studies of Emission from the Sun at Metre Wavelengths, Cambridge Univ. Press, New York, 1985.

[8] A. O. Benz, Plasma Astrophysics, Springer, Dordrecht, 2002.

[9] Z. S. Hamidi, N. Anim, N. N. M. Shariff, Z. Z. Abidin, Z. A. Ibrahim, C. Monstein, Dynamical structure of solar radio burst type III as evidence of energy of solar flares, in: R.Shukor (Ed.), PERFIK 2012, American Institute of Physics, Malaysia, 2013, pp. 11-15.

[10] Z. S.Hamidi, Z. Abidin, Z. Ibrahim, C. Monstein, N. Shariff, International Journal of Fundamental Physical Sciences 2 (2012) 32-34.

[11] Z. S. Hamidi, Z. Ibrahim, Z. Abidin, M. Maulud, N. Radzin, N. Hamzan, N. Anim, N. Shariff, International Journal of Applied Physics and Mathematics 2 (2012) 3.

[12] Z. S.Hamidi, S. Chumiran, A. Mohamad, N. Shariff, Z. Ibrahim, N. Radzin, N. Hamzan, N. Anim, A. Alias, American Journal of Modern Physics 2 (2013) 4.

[13] A. O. Benz, C. Monstein, H. Meyers, CALLISTO, A New Concept for Solar Radio Spectrometer, Kluwer Academic Publishers, The Netherland, 2004.

[14] Z. S. Hamidi, Z. Abidin, Z. Ibrahim, N. Shariff, C. Monstein, Observations of coronal mass ejections (CMEs) at low frequency radio region on 15th April 2012, in: R. Shukor (Ed.), PERFIK 2012, American Institute of Physics, Malaysia, 2013, pp. 5.

[15] Z. S.Hamidi, N. N. M.Shariff, Evaluation of Signal to Noise Ratio (SNR) of Log Periodic Dipole Antenna (LPDA) BEIAC 2013, IEEE, Langkawi, Malaysia, 2013, pp. 6.

[16] Z. S. Hamidi, N. Shariff, Z. Abidin, Z. Ibrahim, C. Monstein, Middle-East Journal of Scientific Research 12 (2012) 6.

[17] Z. S. Hamidi, N. N. M. Shariff, C. Monstein, W. N. A. Wan Zulkifli, M. B. Ibrahim, N. S. Arifin, N. A. Amran, International Letters of Natural Sciences 5 (2014) 10-17.

[18] Z. S. Hamidi, N. N. M. Shariff, International Letters of Chemistry, Physics and Astronomy 4 (2014) 29-36.

[19] Z. S. Hamidi, N. N. M. Shariff, International Letters of Chemistry, Physics and Astronomy 5 (2014) 32-42.

[20] Z. S. Hamidi, N. N. M. Shariff, International Letters of Chemistry, Physics and Astronomy 5 (2014) 43-49.

[21] Z. S. Hamidi, N. N. M. Shariff, International Letters of Chemistry, Physics and Astronomy 7 (2014) 21-29.

[22] Z. S. Hamidi, N. N. M. Shariff, International Letters of Chemistry, Physics and Astronomy 7 (2014) 30-36.

[23] Z. S. Hamidi, N. N. M. Shariff, C. Monstein, Z. A. Ibrahim, International Letters of Chemistry, Physics and Astronomy 7 (2014) 37-44.

[24] Z. S. Hamidi, N. N. M. Shariff, C. Monstein, W. N. A. Wan Zulkifli, M. B. Ibrahim, N. S. Arifin, N. A. Amran, International Letters of Chemistry, Physics and Astronomy 8 (2014) 13-19. 
[25] Z. S. Hamidi, N. N. M. Shariff, C. Monstein, W. N. A. Wan Zulkifli, M. B. Ibrahim, N. S. Arifin, N. A. Amran, International Letters of Chemistry, Physics and Astronomy 9 (2014) 8-15.

[26] Z. S. Hamidi, N. N. M. Shariff, M. F. Ali, C. Monstein, W. N. A. Wan Zulkifli, M. B. Ibrahim, N. S. Arifin, N. A. Amran, International Letters of Chemistry, Physics and Astronomy 9 (2014) 84-92.

( Received 25 February 2014; accepted 02 March 2014 ) 